\title{
Development and evaluation of a prototype non-woven fabric filter for purification of malaria-infected blood
}

\author{
Zhi-Yong Tao ${ }^{1,2,3,4}$, Hui Xia ${ }^{1 \dagger}$, Jun $\mathrm{Cao}^{2,3}$ and Qi Gao ${ }^{2,3,4^{*}}$
}

\begin{abstract}
Background: Many malaria-related studies depend on infected red blood cells (iRBCs) as fundamental material; however, infected blood samples from human or animal models include leukocytes (white blood cells or WBCs), especially difficult to separate from iRBCs in cases involving Plasmodium vivax. These host WBCs are a source of contamination in biology, immunology and molecular biology studies, requiring their removal. Non-woven fabric (NWF) has the ability to adsorb leukocytes and is already used as filtration material to deplete WBCs for blood transfusion and surgery. The present study describes the development and evaluation of a prototype NWF filter designed for purifying iRBCs from malaria-infected blood.
\end{abstract}

Methods: Blood samples of $P$. vivax patients were processed separately by NWF filter and CF11 column methods. WBCs and RBCs were counted, parasite density, morphology and developing stage was checked by microscopy, and compared before and after treatment. The viability of filtrated $P$. vivax parasites was examined by in vitro shortterm cultivation.

Results: A total of 15 P. vivax-infected blood samples were treated by both NWF filter and CF11 methods. The WBC removal rate of the NWF filter method was $99.03 \%$, significantly higher than the CF11 methods $(98.41 \%, P<$ 0.01). The RBC recovery rate of the NWF filter method was $95.48 \%$, also significantly higher than the CF11 method $(87.05 \%, P<0.01)$. Fourteen in vitro short-term culture results showed that after filter treatment, $P$. vivax parasite could develop as normal as CF11 method, and no obvious density, developing stage difference were fund between two methods.

Conclusions: NWF filter filtration removed most leukocytes from malaria-infected blood, and the recovery rate of RBCs was higher than with CF11 column method. Filtrated $P$. vivax parasites were morphologically normal, viable, and suitable for short-term in vitro culture. NWF filter filtration is simple, fast and robust, and is ideal for purification of malaria-infected blood.

\section{Background}

Malaria is a mosquito borne parasitic disease that causes about 1 million fatalities a year, most occurring in Africa. According to a WHO estimate, 3.3 billion people are at risk of malaria [1]. Because no effective vaccine currently exists, all humans are potentially susceptible to malaria. Better control and eradication of malaria

\footnotetext{
* Correspondence: gaoqi54@hotmail.com

† Contributed equally

${ }^{2}$ Jiangsu Institute of Parasitic Diseases, Meiyuan Yangxiang 117, Wuxi 214064, People's Republic of China

Full list of author information is available at the end of the article
}

requires more thorough research on the biological characteristics of the parasite.

The establishment of continuous cultures of Plasmodium falciparum provided researchers with abundant materials that led to several breakthroughs [2]. Compared to $P$. falciparum, research on Plasmodium vivax is less extensive, not only because vivax malaria is relative mild and therefore neglected, but also because of the biological properties of P. vivax[3]. First, in vitro cultivation of $P$. vivax is not well established, and limited to short-term maintenance. Usually, parasite density of $P$. vivax during culture does not obviously increase as it does with P. falciparum, but decreases [4]. Second,

\section{Biomed Central}


the animal model of $P$. vivax is endangered non-human primates, which are difficult to obtain. The biological properties of in vivo propagated parasites are not exactly the same as parasites isolated from naturally infected humans, so they cannot represent the entire set of characteristics of $P$. vivax in nature. Rodent malaria parasites are easy to propagate in vivo, and parasitaemia can be high, making it a realistic animal model for some malaria researches [5]. However, blood from infected human or animal models includes leukocytes, and especially in cases of $P$. vivax cases, the amount of WBCs is often equal to or greater than iRBCs. These host source WBCs have high metabolism capacity, are a contamination in malaria biology, immunology and molecular biology studies, and need to be removed [6].

Currently, the methods for removing WBCs from malaria-infected blood include CF11 column and Plasmodipur filter filtration [7-13]. CF11 cellulose column is the most commonly used method, it is cheap but time consuming and laborious, and Plasmodipur filter is expensive for resource-limited settings, therefore a new method is required for purification of malaria-infected blood. Leukocytes can be depleted by filtering through non-woven fabric filters [14], because this type of blood cells had strong adhesion function based on its cell surface properties. This is in contrast to RBCs, which are capable of deformation, and can escape from the rigid fibers [15]. Even though the surface of iRBCs is altered by parasite protein trafficking to the host cell membranes, iRBCs still flow through the filter material, allowing iRBCs to be purified from WBC contaminants. The present study describes the development and evaluation of a prototype NWF filter designed for purifying iRBCs from malaria-infected blood.

\section{Methods}

\section{The development of prototype NWF filter}

The PBT (polybutylene terephthalate) melt-blown nonwoven fabric modified with polar groups(Gaolite medical equipment Ltd., Zhangjiagang, China) was used as the filter media, and the shape of NWF filter was referred to single-use sterilizing filter. Injection molding machine (MA1200/370, Haitian, Ningbo, China) and ultrasonic plastic welder (EM20-2, Branson, Shanghai, China) were used to produce and assemble the polycarbonate shells with NWF filter pad.

There are four major criteria of new filter for purification of malaria-infected blood: a) One single-use filter is capable for treatment of $5 \mathrm{ml} P$. vivax-infected blood; b) For $5 \mathrm{ml}$ whole blood, the WBC removal rate should be $>99 \%$; c) After filtration, malaria parasite keeps viable, and is suitable for in vitro short-term culture; d) It is also suitable for treatment of rodent malaria-infected blood.

\section{Plasmodium vivax-infected sample collection and processing}

Plasmodium vivax-infected blood samples were collected in the north part of Anhui province from July 2007 to September 2010. Only P. vivax was prevalent in this area [16]. All malaria cases were confirmed by microscopy. After consent was obtained from adult patients, $10-15 \mathrm{ml}$ of whole blood was drawn into heparin-treated vacuum tubes, and mixed. Blood samples were sealed and immersed in $37^{\circ} \mathrm{C}$ water in a thermos flask and immediately transferred to the laboratory in the Parasitology Department of Bengbu Medical College. Thick- and thinsmear were made, stained with Giemsa (Fisher, USA), and examined by microscopy. After mixing and transferring whole blood into a $50 \mathrm{ml}$ centrifuge tube, and spinning for $5 \mathrm{~min}$ at 2,000 rpm, plasma was removed. To compare efficiency of leukocyte removal, incomplete RPMI 1640 medium (Invitrogen, USA) was used to resuspend the cell pellet to $30 \mathrm{ml}$. A $200 \mu \mathrm{l}$ cell suspension was transferred to a centrifuge tube, for counting WBCs and RBCs using the improved Neubauer chamber method. A $20 \mathrm{ml}$ cell suspension was separated in two 50 $\mathrm{ml}$ centrifuge tubes, each containing the equivalent of about $5 \mathrm{ml}$ of whole blood.

\section{Purification of $P$. vivax-infected blood sample by the NWF filter method}

Ten $\mathrm{ml}$ of cell suspension prepared above was sucked into a $20 \mathrm{ml}$ syringe, the filter inlet interface was adapted to the syringe, and the plunger was gently pushed (about $5 \mathrm{ml} / \mathrm{min}$ ). Then collect flow-through cell suspension from the filter outlet into a new $50 \mathrm{ml}$ centrifuge tube. The filter was washed with $5 \mathrm{ml}$ RPMI 1640, and eluted cell suspension collected in the same tube. The sample was spun $10 \mathrm{~min}$ at 3,000 $\mathrm{rpm}$, the supernatant discarded, and RPMI 1640 used to resuspend the pellet to $10 \mathrm{ml}$, and WBCs and RBCs were counted as described above. A $10 \mu \mathrm{l}$ cell suspension was used to make thickand thin-smears for staining and examination as above.

\section{Purification of $P$. vivax-infected blood sample by CF11 column method}

CF11 powder was purchased from Whatman (Maidstone, UK), and CF11 columns were prepared as described previously [7]. The CF11 powder bed was packed in a central luer syringe column with a sterile foil package by tapping on the bench, and a pre-rinsed CF11 bed was made by dropping in $5 \mathrm{ml}$ RPMI 1640. Before the last portion of liquid ran into the bed, $10 \mathrm{ml}$ of cell suspension was applied to the bed dropwise, and then the bed was rinsed with $5 \mathrm{ml}$ RPMI 1640. The cell suspension was eluted into a $50 \mathrm{ml}$ centrifuge tube. Sample washing, counting of WBCs and RBCs, slide 
making and checking were as above. And the results were compared to NWF filter method.

\section{In vitro short-term culture of filtrated $P$. vivax samples} Both NWF filter and CF11 column treated RBC samples from $P$. vivax patients with more than $50 \%$ ring stage parasites and without prior anti-malarial therapy were washed three times with RPMI 1640, and resuspended at 2\% haematocrit in McCoy's 5A medium (SigmaAldrich) containing 20\% human $\mathrm{AB}+$ serum (prepared from malaria-naive donors)[17] and added into 10 wells of a 96-well plate, each well contains $100 \mu \mathrm{l}$ of cell suspension. The test plate was incubated at $37^{\circ} \mathrm{C}, 5 \% \mathrm{CO}_{2}$, humidified environment for $48 \mathrm{~h}$. A thick- and thinsmear was made to check the development status of $P$. vivax parasites for every 8 hours. The parasitaemia and morphology of parasite of each slide were checked by microscopy.

\section{Purification of $P$. berghei-infected mouse blood samples} Heparin-treated Plasmodium berghei-infected mouse blood samples were provided by the Parasitology Department of Bengbu Medical College. Plasma of each sample was removed by centrifugation, and packed cells were diluted in RPMI 1640 to $5 \mathrm{ml}$, then filtered through NWF filter and washed as previously for $P$. vivax samples. Sampling and counting for WBCs were carried out as above. Thick- and thin-smears were made and Giemsa stained pre- and post filtration for observation.

\section{Purification of $P$. falciparum sample in vitro}

A $0.5 \mathrm{ml}$ of in vitro-cultured P. falciparum (FCC1/HN isolate) sample at 5\% parasitaemia was mixed with fresh prepared $4.5 \mathrm{ml}$ of healthy volunteer's plasma-free blood, and then filtered through NWF filter as described above. Thick- and thin- smears were made and Giemsa stained to observe the change of WBCs and infected RBCs pre- and post filtration.

\section{Statistical analysis}

All data were entered and analysed using SPSS for Windows v14 (SPSS Inc., Chicago, IL). The Wilcoxon two-sample paired signed rank test was used to compare the differences in $\mathrm{WBC}$ removal, $\mathrm{RBC}$ recovery and parasite density between NWF filter and CF11 column methods. Statistical significance was set at $P \leq$ 0.05 .

\section{Ethical considerations}

The study was approved by the Institutional Review Board (IRB00004221) of Jiangsu Institute of Parasitic Diseases, Wuxi, China.

Questionnaire surveys, physical examination and laboratory work were conducted after the purpose of the study had been explained to participants, who were given the right to withdraw from the study at any time, without consequences. Written informed consent was obtained from each participant.

\section{Results}

Development of NWF filter for purification of malariainfected blood

Upper and lower parts of polycarbonate shells of new NWF filter were produced by plastic injection molding. It has three layers of non-woven fabric unit in $2.2 \mathrm{~cm}$ diameter as filter pad, and ultrasonic welding method was adopted for assembling the upper part, filter pad and lower part into a prototype NWF filter (Figure 1). Filters were washed by distilled water and sterilized with ethylene oxide gas, and stored in GMP environment for one month before use.

\section{Comparison of NWF filter filtration and CF11 column methods for purification of $P$. vivax-infected blood}

A total of 15 P. vivax-infected blood samples were used for both NWF filtration and CF11 methods. WBCs and RBCs were counted before and after treatment, and the removal rate of $\mathrm{WBCs}$ and the recovery rate of $\mathrm{RBCs}$ were calculated, and the parasite density was counted by microscopy (Additional file 1). The average WBC removal rate by the NWF filter was $99.03 \%$, and the RBC recovery rate was $95.48 \%$. The average $\mathrm{WBC}$ removal rate of the CF11 method was $98.41 \%$, and the RBC recovery rate was $87.05 \%$. Significance differences were observed between the two methods for WBC removal $(P<0.01)$, and a clear significant difference between the two methods was seen for RBC recovery $(P<0.01)$. The NWF filter method was better than the CF11 column method, especially for high performance $\mathrm{RBC}$ recovery. Post-filtration, there is no parasite density difference between NWF filter and CF11column methods $(P>0.05)$ by microscopy, but the WBCs were substantially reduced (Figure 2).

\section{In vitro short-term culture of $P$. vivax after filtration}

A total of 14 P. vivax-infected blood samples were treated by both NWF filter filtration and CF11 column methods, and subjected to in vitro short-term culture. Compared to CF11 column method, parasites filtrated by NWF filter are of same ability to continue developing into mature stage in vitro, and there are no obvious changes been observed in parasite density, morphology or stage proportion between two methods by microscopy (Figure 3).

\section{Purification of $P$. berghei-infected sample}

Five $P$. berghei-infected mouse blood samples were diluted and filtrated by NWF filter method, the WBC removal rate was $99.21 \%$. By microscopy comparison of 


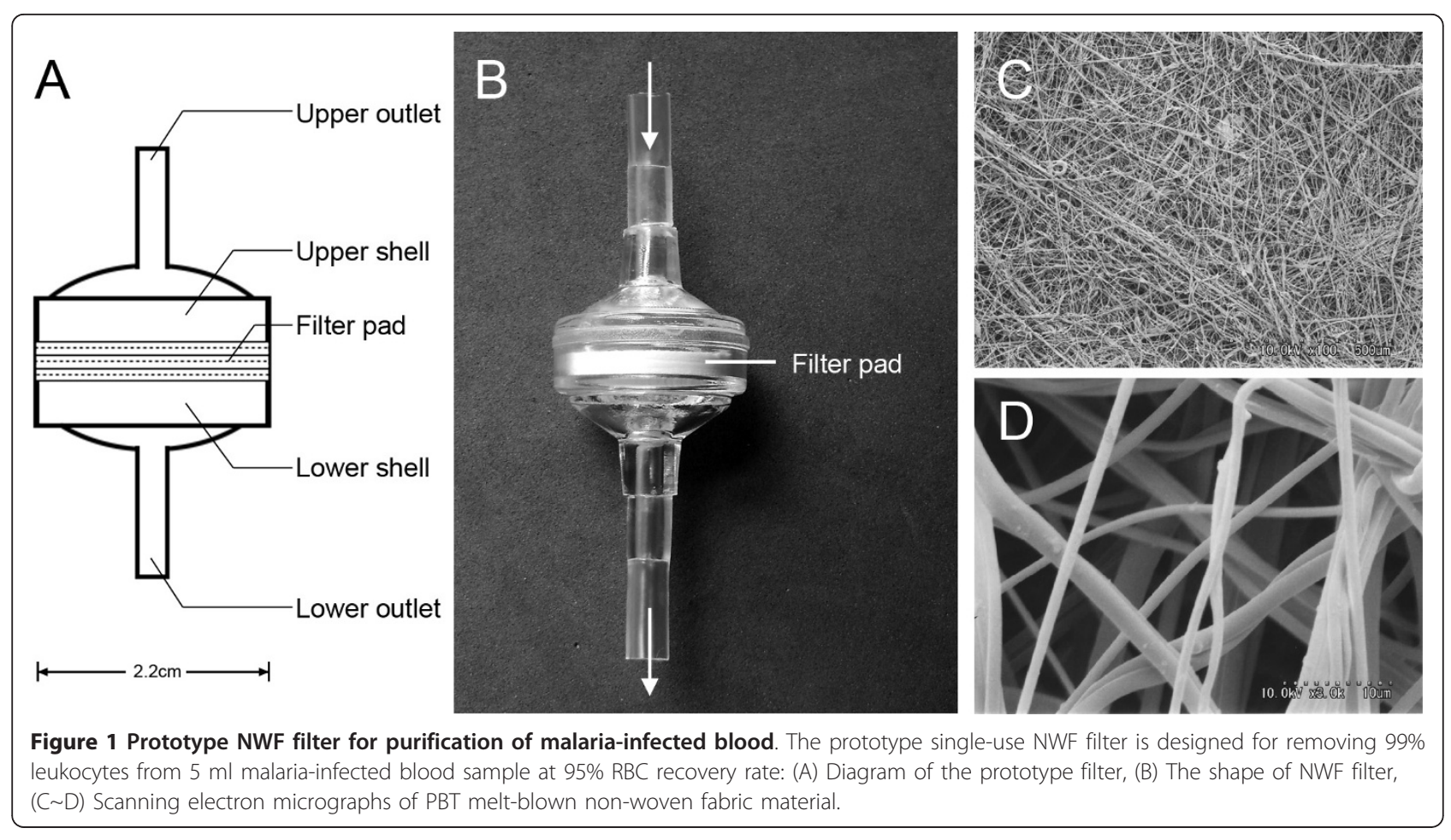

pre- and post filtration smears: there are no obvious parasite density, morphology or stage proportion changes of $P$. berghei been observed (Figure 4).

\section{Purification of $P$. falciparum in vitro}

When the WBCs were removed from the sample mixed with in vitro cultured P. falciparum-infected RBCs and healthy donor blood, there were no obvious changes in parasite density, morphology or stage proportion of $P$. falciparum observed between pre- and post-filter filtration.

\section{Discussion}

The prototype NWF filter developed here was designed to remove $>99 \%$ of leukocytes from $5 \mathrm{ml}$ of whole blood, with a 95\% RBC recovery rate. After testing with 15 P. vivax-infected blood samples, the rates of WBC removal and RBC recovery for the NWF filter method were $99.03 \%$ and $95.48 \%$, and for the CF11 method they were $98.41 \%$ and $87.05 \%$. NWF filter showed both higher WBC removal and RBC recovery rates over CF11 column method. To save time in sample processing, only one wash step was used, which may have contributed to the difference in $\mathrm{RBC}$ recovery between the two methods.

In some studies, the purity and viability of parasites in malaria samples are crucial for results, and a convenient method for removal of contaminating WBCs is needed. In this study, processing $5 \mathrm{ml}$ of whole blood using the CF11 method requires at least 30 minutes from pre-rinsing the column bed to the final drop of the cell suspension flowthrough. In contrast, the NWF filter method saves time and is easy to perform. Filtration of same amount blood is possible in 5 minutes from diluting sample to the end of the wash procedure. Furthermore, the filtration method is more robust than the CF11 method; the latter method may fail if the column was not prepared properly, resulted in an unacceptable amount of WBCs leakage. NWF filters could be sealed in plastic bag and batch sterilized thus could be ready to use, with no need to prepare or autoclave before use, as is required for CF11 columns. Training for using NWF filter is minimal, and researchers can successfully perform filtration by following the protocol. Filter filtration does not need additional equipment, so it is suitable for use in field settings.

According to malaria genome and transcriptome studies, contamination from the host is the difficult problem in research, lowering the credibility of sequencing and annotation results [18]. For example, about $10 \%$ of host sequences are incorrectly in the Plasmodium yoelii genome results [19]. By NWF filtration, more than 99\% leukocytes were removed from malaria-infected blood, providing purified materials for downstream malaria research, it suggest that NWF filter could be useful for sample pretreatment in malaria molecular studies.

In recent years, reports on the chloroquine resistance of $P$. vivax have gradually increased, and in vitro tests were considered as direct and more important evidence 


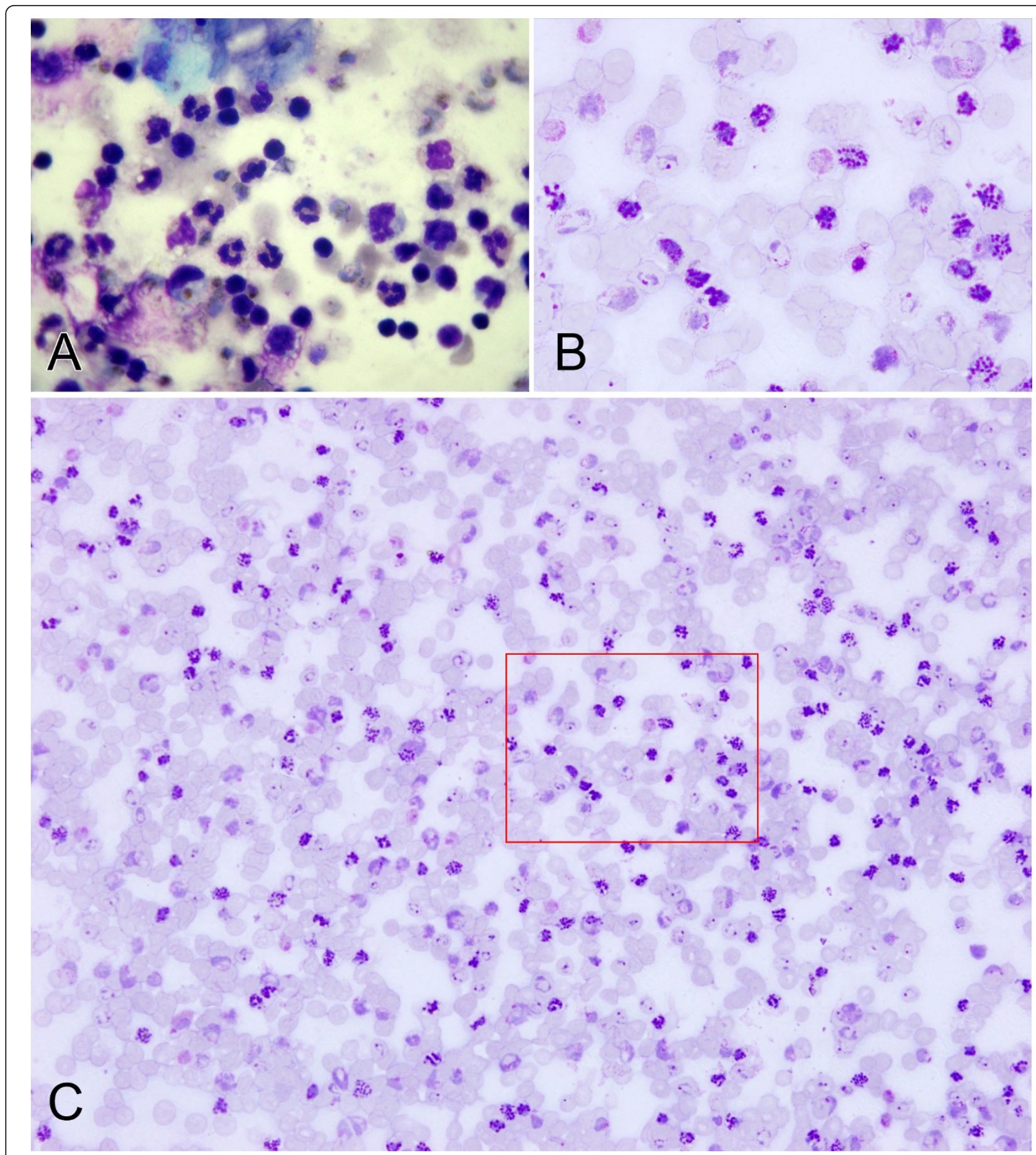

Figure 2 Leukocyte removal efficacy of NWF filter filtration coupled with $60 \%$ Percoll concentration. A $P$. vivax blood sample was treated by NWF filter filtration then performed 60\% Percoll concentration: (A) Excessive WBC contamination in the 60\% Percoll enrichment slide without removing WBC (100x oil-immersion objective), (B) With NWF filter filtration, no WBCs could be observed. (100x oil-immersion objective), (C) Even including more RBCs, still no single WBC was fund (40x high power objective), the rectangle represent the same field of (B).

[20]. The existence of WBCs in short-term cultures of $P$. vivax not only disturbs the development of parasites and interferes with microscopy, more importantly, makes drug accumulated and decreases the concentration of drugs, and so $\mathrm{IC}_{50}$ results may be falsely achieved [21]. Thus, removal of WBCs from $P$. vivax-infected blood samples is a necessary procedure for in vitro drug susceptibility tests. In this study, $P$. vivax parasites filtered 


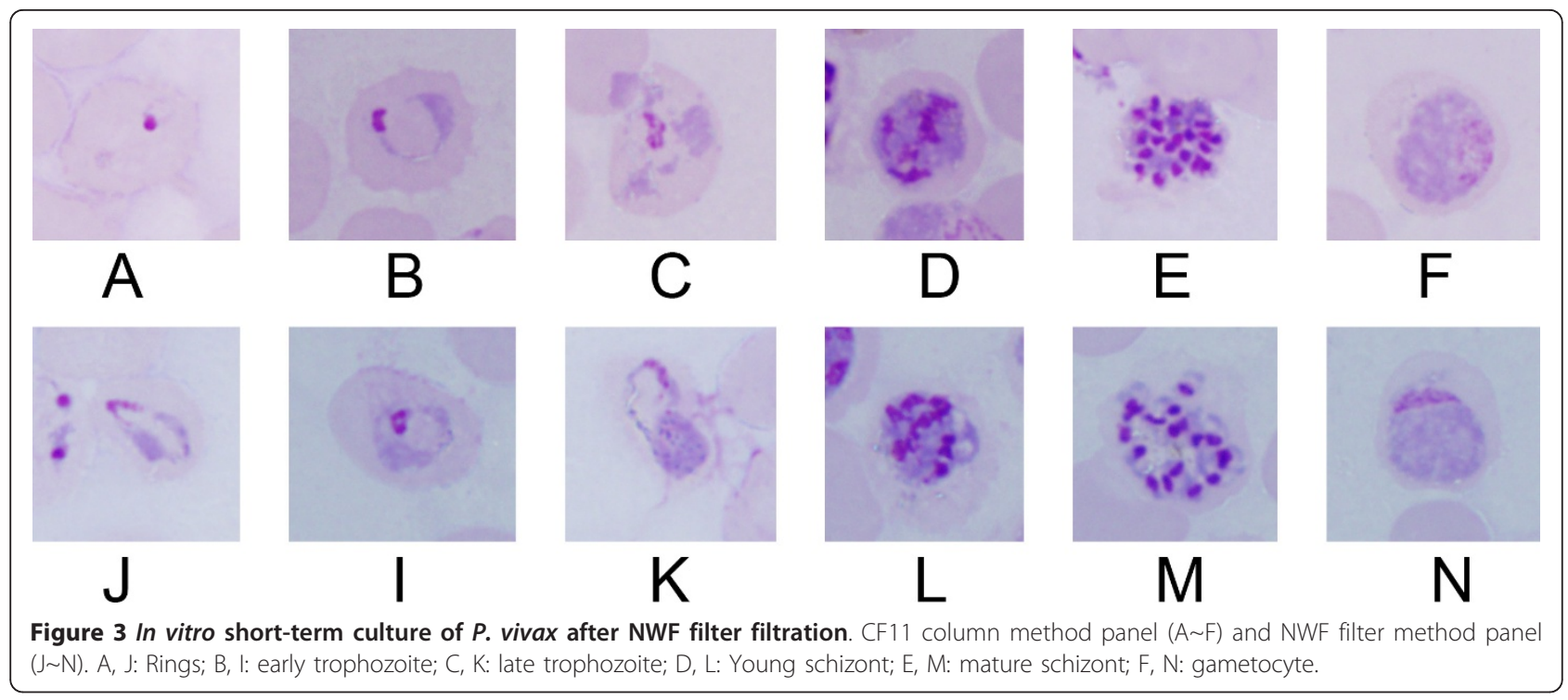

by NWF filter were successfully short-term cultured in vitro, and with no obvious changes in density, morphology and stage proportion compared to CF11 method. Escaped WBCs and platelets may cast negative impact on $P$. vivax during in vitro culture, and post NWF filter treatment, the major component of escaped WBCs were lymphocytes, and most of platelets were removed, these properties were similar to CF11 method [7]. The development of NWF filter provides researchers a useful tool for further in vitro antimalarial drug sensitivity surveillance.

Using rodent models to investigate malaria has obvious advantages including easy for maintaining, cost efficient and avoiding risks associated with human material. In this study, results showed NWF filter are suitable for purification of $P$. berghei-infected mouse blood, among high WBC removal ability, more importantly, the filter method enables a higher $\mathrm{RBC}$ recovery rate than the CF11 method, making it more convenient for treatment of small amount samples. Even in vitro cultivation could provide purified P. falciparum parasites, though the parasites isolated from patient blood still play unique role in many studies. The new NWF filter could be used for purification of $P$. falciparum samples too. In this study, including mature stage $P$. falciparum-infected $\mathrm{RBC}$ could pass through the non-woven fabric filter material, this result is different from the report of using routine transfusion filter to deplete $P$. falciparuminfected RBC [22], this may be caused by the difference of filtration materials, sample size and iRBC load.

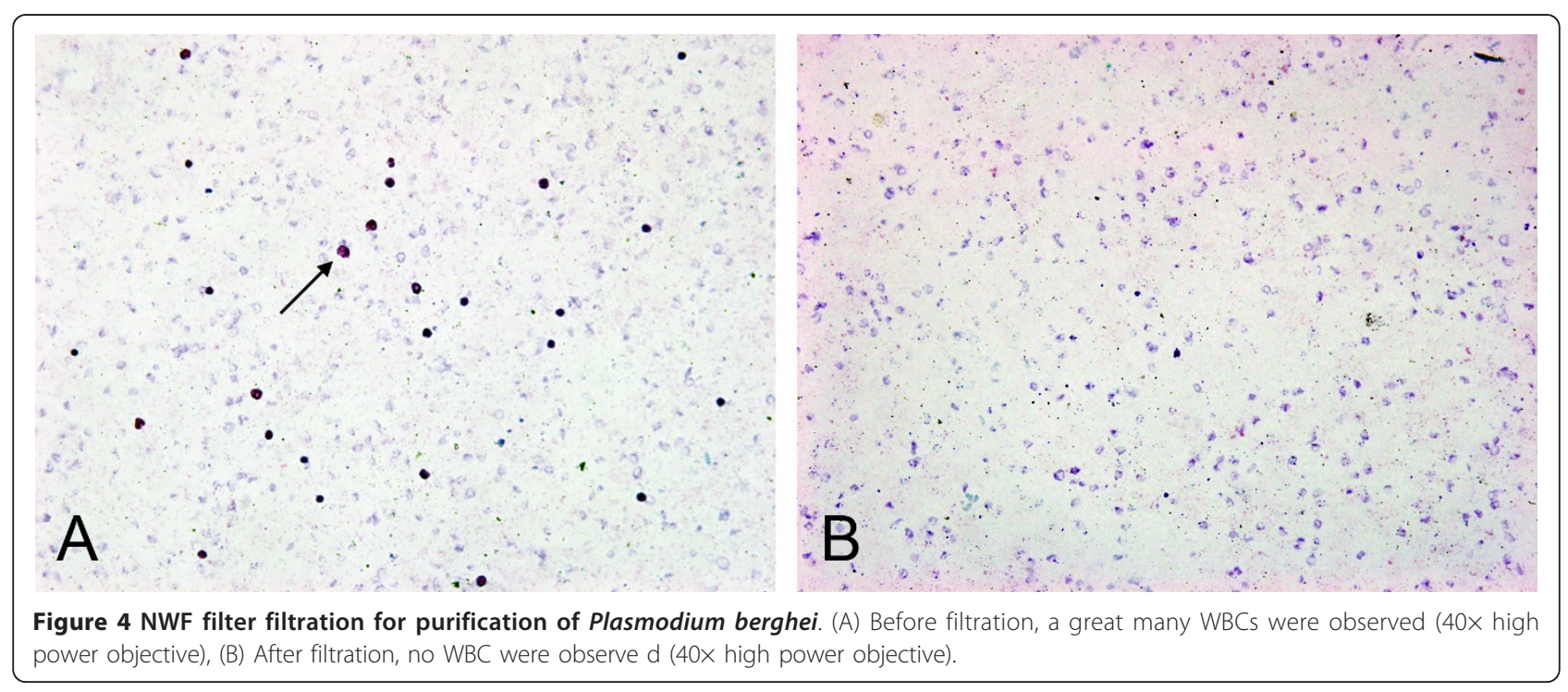




\section{Conclusions}

NWF filter filtration removed most leukocytes from malaria-infected blood, and the recovery rate of RBCs was higher than with CF11 column method. Filtration could enable more efficient use of malaria samples for research. No obvious parasite density decrease or morphology changes were observed, and the viability of parasites was also well preserved during processing. P. vivax blood samples filtered by NWF filter were successfully used in short-term in vitro cultivation. It is also suitable for purifying P. berghei-infected mouse blood and P. falciparuminfected human blood. A new prototype NWF filter was successfully developed, and the NWF filter filtration method is simple, fast and robust, and ideal for purification of malaria-infected blood.

\section{Additional material}

Additional file 1: The efficacy comparison results of NWF filter filtration and CF11 column methods for purifying Plasmodium vivax-infected blood. The WBC, RBC and parasite density results of Plasmodium vivax-infected blood samples before and post treatment by NWF filter filtration and CF11 column methods.

\begin{abstract}
Acknowledgements
This work was supported by grants from the National Natural Science Foundation of China (No. 30872214), the Asia Pacific Malaria Elimination Network Establishment Support Programme (No. 107-02), the National Basic Research Programme (973 Programme) in China (2007CB513100), the National S \& T Major Programme (Grant No. 2008ZX10004-011), and the Open Programme of Key Laboratory on Technology for Parasitic Disease Prevention and Control of Chinese Ministry of Health (No. WK009-001).
\end{abstract}

\section{Author details}

'Department of Parasitology, Bengbu Medical College, 2600 Donghai Dadao Road, Bengbu 233030, People's Republic of China. ${ }^{2}$ Jiangsu Institute of Parasitic Diseases, Meiyuan Yangxiang 117, Wuxi 214064, People's Republic of China. ${ }^{3}$ Key Laboratory on Technology for Parasitic Disease Prevention and Control, Ministry of Health, Meiyuan Yangxiang 117, Wuxi 214064, People's Republic of China. ${ }^{4}$ Department of Parasitology, Medical College of Soochow University, Suzhou 215123, People's Republic of China.

\section{Authors' contributions}

ZYT, JC and QG conceived the study and participated in its design and coordination. ZYT carried out NWF filter designing and comparison of filter filtration and CF11 method. HX contributed expertise in field sample collection, in vitro short-term culture. ZYT wrote the manuscript. JC, HX helped on results analysis and manuscript drafting. All authors read and approved the final manuscript.

\section{Competing interests}

The authors declare that they have no competing interests.

Received: 15 April 2011 Accepted: 25 August 2011

Published: 25 August 2011

\section{References}

1. WHO: World malaria report 2008 Geneva, World Health Organization; 2008.

2. Trager $W$, Jensen JB: Human malaria parasites in continuous culture. Science 1976, 193:673-675.

3. Baird JK: Neglect of Plasmodium vivax malaria. Trends Parasitol 2007, 23:533-539.
4. Udomsangpetch R, Kaneko O, Chotivanich K, Sattabongkot J: Cultivation of Plasmodium vivax. Trends Parasitol 2008, 24:85-88.

5. Stewart VA: Plasmodium vivax under the microscope: the Aotus model. Trends Parasitol 2003, 19:589-594.

6. McKenzie FE, Prudhomme WA, Magill AJ, Forney JR, Permpanich B, Lucas C, Gasser RA Jr, Wongsrichanalai C: White blood cell counts and malaria. J Infect Dis 2005, 192:323-330.

7. Sriprawat K, Kaewpongsri S, Suwanarusk R, Leimanis ML, Lek-Uthai U, Phyo AP, Snounou G, Russell B, Renia L, Nosten F: Effective and cheap removal of leukocytes and platelets from Plasmodium vivax infected blood. Malar J 2009, 8:115.

8. Barnwell JW, Nichols ME, Rubinstein P: In vitro evaluation of the role of the Duffy blood group in erythrocyte invasion by Plasmodium vivax. J Exp Med 1989, 169:1795-1802.

9. Suwanarusk $R$, Chavchich $M$, Russell $B$, Jaidee A, Chalfein F, Barends $M$, Prasetyorini B, Kenangalem E, Piera KA, Lek-Uthai U, Anstey NM, Tjitra E, Nosten F, Cheng Q, Price RN: Amplification of pvmdr1 associated with multidrug-resistant Plasmodium vivax. J Infect Dis 2008 , 198:1558-1564.

10. Bozdech Z, Mok S, Hu G, Imwong M, Jaidee A, Russell B, Ginsburg H, Nosten F, Day NP, White NJ, Carlton JM, Preiser PR: The transcriptome of Plasmodium vivax reveals divergence and diversity of transcriptional regulation in malaria parasites. Proc Natl Acad Sci USA 2008, 105:16290-16295.

11. Handayani S, Chiu DT, Tjitra E, Kuo JS, Lampah D, Kenangalem E, Renia L, Snounou G, Price RN, Anstey NM, Russell B: High deformability of Plasmodium vivax-infected red blood cells under microfluidic conditions. J Infect Dis 2009, 199:445-450.

12. Rottmann M, McNamara C, Yeung BK, Lee MC, Zou B, Russell B, Seitz P, Plouffe DM, Dharia NV, Tan J, Cohen SB, Spencer KR, González-Páez GE, Lakshminarayana SB, Goh A, Suwanarusk R, Jegla T, Schmitt EK, Beck HP, Brun R, Nosten F, Renia L, Dartois V, Keller TH, Fidock DA, Winzeler EA, Diagana $\Pi$ : Spiroindolones, a potent compound class for the treatment of malaria. Science 2010, 329:1175-1180.

13. Janse CJ, Camargo A, Del Portillo HA, Herrera S, Kumlien S, Mons B, Thomas A, Waters AP: Removal of leucocytes from Plasmodium vivaxinfected blood. Ann Trop Med Parasitol 1994, 88:213-216.

14. Kim EJ, Yeo GD, Pai CM, Kang IK: Preparation of surface-modified poly (butylene terephthalate) nonwovens and their application as leukocyte removal filters. J Biomed Mater Res B Appl Biomater 2009, 90:849-856.

15. Matheis G, Moritz A, Scholz M: Leukocyte depletion in cardiac surgery and cardiology Basel, Karger; 2002, 18-19.

16. Zhang W, Wang L, Fang L, Ma J, Xu Y, Jiang J, Hui F, Wang J, Liang S, Yang H, Cao W: Spatial analysis of malaria in Anhui province, China. Malar J 2008, 7:206.

17. Russell BM, Udomsangpetch R, Rieckmann KH, Kotecka BM, Coleman RE, Sattabongkot J: Simple in vitro assay for determining the sensitivity of Plasmodium vivax isolates from fresh human blood to antimalarials in areas where $P$. vivax is endemic. Antimicrob Agents Chemother 2003, 47:170-173.

18. Deitsch KW, Carlton JM, Wootton JC, Wellems TE: Host sequences in Plasmodium falciparum and Plasmodium vivax genomic DNA: horizontal transfer or contamination artifact? FEBS Lett 2001, 491:164-165.

19. Carlton JM, Angiuoli SV, Suh BB, Kooij TW, Pertea M, Silva JC, Ermolaeva MD, Allen JE, Selengut JD, Koo HL, Peterson JD, Pop M, Kosack DS, Shumway MF, Bidwell SL, Shallom SJ, van Aken SE, Riedmuller SB, Feldblyum TV, Cho JK, Quackenbush J, Sedegah M, Shoaibi A, Cummings LM, Florens L, Yates JR, Raine JD, Sinden RE, Harris MA, Cunningham DA, Preiser PR, Bergman LW, Vaidya AB, van Lin LH, Janse CJ, Waters AP, Smith HO, White OR, Salzberg SL, Venter JC, Fraser CM, Hoffman SL, Gardner MJ, Carucci DJ: Genome sequence and comparative analysis of the model rodent malaria parasite Plasmodium yoelii yoelii. Nature 2002, 419:512-519.

20. Baird JK: Resistance to therapies for infection by Plasmodium vivax. Clin Microbiol Rev 2009, 22:508-534.

21. Kaewpongsri $S$, Sriprawat $K$, Suwanarusk R, Kyle DE, Lek-Uthai $U$, Leimanis M, Lwin KM, Phyo AP, Zwang J, Russell B, Nosten F, Renia L: Presence of leukocytes in ex vivo assays significantly increase the $I C_{50}$ of artesunate and chloroquine against $P$. vivax and $P$. falciparum. Antimicrob Agents Chemother 2010, 55:1300-1304. 
22. Cardo L, Salata J, Wilder D: Removal of Plasmodium falciparum-infected red blood cells from whole blood by leukoreduction filters. Transfusion 2009, 49:337-346.

doi:10.1186/1475-2875-10-251

Cite this article as: Tao et al: Development and evaluation of a

prototype non-woven fabric filter for purification of malaria-infected

blood. Malaria Journal 2011 10:251.

Submit your next manuscript to BioMed Central and take full advantage of:

- Convenient online submission

- Thorough peer review

- No space constraints or color figure charges

- Immediate publication on acceptance

- Inclusion in PubMed, CAS, Scopus and Google Scholar

- Research which is freely available for redistribution

Submit your manuscript at www.biomedcentral.com/submit 